\title{
Bases cerebrales de la atención sostenida y la memoria de trabajo: un estudio de resonancia magnética funcional basado en el Continuous Performance Test
}

\author{
Manel Bartés-Serrallonga, Ana Adan, Jordi Solé-Casals, Xavier Caldú, Carles Falcón, Montserrat Pérez-Pàmies, \\ Núria Bargalló, Josep M. Serra-Grabulosa
}

Introducción. Uno de los paradigmas más utilizados en el estudio de la atención es el Continuous Performance Test (CPT).
La versión de pares idénticos (CPT-IP) se ha utilizado ampliamente para evaluar los déficits de atención en los trastornos
del neurodesarrollo, neurológicos y psiquiátricos. Sin embargo, la localización de la activación cerebral de las redes aten-
cionales varía significativamente según el diseño de resonancia magnética funcional (RMf) usado.

Objetivo. Diseñar una tarea para evaluar la atención sostenida y la memoria de trabajo mediante RMf para proporcionar datos de investigación relacionados con la localización y el papel de estas funciones.

Sujetos y métodos. El estudio contó con la participación de 40 estudiantes, todos ellos diestros (50\%, mujeres; rango: 18-25 años). La tarea de CPT-IP se diseñó como una tarea de bloques, en la que se combinaban los períodos CPT-IP con los de reposo.

Resultados. La tarea de CPT-IP utilizada activa una red formada por regiones frontales, parietales y occipitales, y éstas se relacionan con funciones ejecutivas y atencionales.

Conclusiones. La tarea de CPT-IP utilizada en nuestro trabajo proporciona datos normativos en adultos sanos para el estudio del sustrato neural de la atención sostenida y la memoria de trabajo. Estos datos podrían ser útiles para evaluar trastornos que cursan con déficits en memoria de trabajo y en atención sostenida.

Palabras clave. Atención sostenida. Corteza parietal. Corteza prefrontal. CPT-IP. Memoria de trabajo. RMf.

\section{Introducción}

Los estudios clínicos y en población sana han mejorado el conocimiento sobre las redes neurales implicadas en la atención [1]. Desde un punto de vista neurológico, las redes atencionales se pueden dividir en tres componentes principales [2]. El primero corresponde al llamado sistema de alerta o excitación, y es responsable de mantener un estado de receptividad a los estímulos, contribuyendo también a la preparación de las respuestas. Se localiza subcorticalmente, y está formado por el tálamo y la formación reticular ascendente, desde la cual se originan proyecciones difusas al sistema límbico y al neocórtex. El segundo componente o sistema de orientación atencional permite la orientación espacial y la ubicación de los estímulos con el fin de seleccionar la información pertinente. Anatómicamente, este sistema está formado por una red corticosubcortical mixta que incluye el núcleo lateral pulvinar del tálamo, el colículo superior y la corteza parietal posterior. El tercer componente es el sistema atencional ejecutivo o el sistema de la atención selectiva, res- ponsable del reclutamiento y el control de las áreas del cerebro necesarias para llevar a cabo funciones cognitivas complejas, así como para regular la dirección y el propósito de la acción. Este tercer componente está situado a nivel cortical y es el principal sustrato anatomofuncional de la atención sostenida.

La atención sostenida se define como la capacidad de mantener un estado de supervisión adecuada a ciertos eventos o estímulos durante largos períodos. Numerosos estudios de neuroimagen centrados en el estudio de las bases cerebrales de la atención sostenida han localizado éstas, principalmente, en las cortezas prefrontal y parietal superior [3]. Específicamente, se ha observado que las regiones frontales (incluyendo la corteza cingulada anterior) participan en el control ejecutivo y la detección de estímulos [4], mientras que las regiones frontal derecha y parietales bilaterales están involucradas en el mantenimiento de la atención de una manera sostenida [5,6]. El tálamo y la formación reticular forman una vía secundaria que contribuye a la realización de tareas a través del control de la activación cortical [7].
Grup de Tecnologies Digitals; Universitat de Vic; Vic, Barcelona (M. Bartés-Serrallonga, J. SoléCasals). Departamento de Psiquiatría y Psicobiología Clínica; Universitat de Barcelona (A. Adan, X. Caldú, M. Pérez-Pàmies J.M. SerraGrabulosa). Institut d'Investigació en Cervell, Cognició i Conducta, IR3C (A. Adan, X. Caldú, J.M. SerraGrabulosa). CIBER-BBN (C. Falcón). Institut d'Investigacions Biomèdiques August Pi i Sunyer, IDIBAPS (C. Falcón, N. Bargalló, J.M. Serra-Grabulosa). Sección de Neurorradiología; Servicio de Radiología; Centre de Diagnòstic per la Imatge, CDI; Hospital Clínic (N. Bargalló). Barcelona, España.

Correspondencia: Dr. Josep M. Serra Grabulosa. Departamento de Psiquiatría y Psicobiología Clínica. Universitat de Barcelona. Pg. Vall d'Hebron, 171 E-08035 Barcelona.

E-mail:

jmserra@ub.edu

Financiación:

Este trabajo ha contado con una subvención del Ministerio Español de Educación y Ciencia (SEJ200508704) y el Departamento Catalán de Universidades e Innovación (2009BE-200239) a J.M.S.G., así como una subvención del gobierno catalán (2010BE1-00772) a J.S.C. y de la Universitat de Vic (R0904).

Aceptado tras revisión externa: 09.12.13.

Cómo citar este artículo: Bartés-Serrallonga $M$, Adan A, Solé-Casals J, Caldú X, Falcón C, Pérez-Pàmies $M$, et al. Bases cerebrales de la atención sostenida y la memoria de trabajo: un estudio de resonancia magnética funcional basado en el Continuous Performance Test. Rev Neurol 2014; 58: 289-95.

English version available at www.neurologia.com (c) 2014 Revista de Neurología 
Entre las diversas pruebas que se utilizan para evaluar la atención sostenida, el Continuous Performance Test-identical pairs (CPT-IP) disfruta de una amplia aceptación en la actualidad [8]. El CPT-IP es una tarea de detección visual que implica la atención sostenida y la memoria de trabajo. Esta tarea fue inicialmente diseñada para detectar los déficits atencionales en pacientes con diagnóstico de esquizofrenia o depresión. Posteriormente, se ha utilizado para estudiar los déficits cognitivos en patologías como el trastorno bipolar [9], la enfermedad de Alzheimer [10,11], la enfermedad de Parkinson [12], la discalculia $[13,14] \mathrm{y}$, especialmente, el trastorno por déficit de atención/hiperactividad (TDAH) [15, 16]. Sin embargo, la localización específica y la distribución relativa de las activaciones de las redes identificadas con resonancia magnética funcional durante la ejecución del CPT varían significativamente según el diseño de la tarea $[17,18]$. Según nuestro conocimiento, sólo existe un estudio de neuroimagen que estudie los patrones de activación cerebral asociados al CPT-IP utilizando estímulos numéricos en sujetos sanos [19]. Además, en este estudio se utilizaron imágenes SPECT, las cuales tienen una menor resolución espacial y temporal que las imágenes de resonancia magnética funcional (RMf).

El objetivo de nuestro estudio es diseñar una tarea para evaluar el sustrato neural de la atención sostenida y la memoria de trabajo, mediante la RMf, que sirva como herramienta para el estudio de las alteraciones cerebrales funcionales en trastornos en los que haya una disfunción de éstas.

\section{Sujetos y métodos}

\section{Muestra}

Para la realización del estudio se reclutó una muestra de 40 estudiantes universitarios voluntarios de la Universidad de Barcelona (50\%, mujeres; rango: 18-25 años; edad media: 19,6 \pm 1,7 años; todos ellos diestros). Se consideraron criterios de exclusión padecer una enfermedad crónica o algún trastorno del sistema nervioso, estar tomando medicación, tener antecedentes de enfermedad mental, así como ser consumidor regular de alcohol y otras drogas. Los participantes no podían tomar ninguna bebida que contuviese cafeína durante un mínimo de $12 \mathrm{~h}$ antes del inicio de la sesión de RMf, ya que la cafeína influye en el rendimiento [20] y en la señal BOLD [21]. El estudio fue aprobado por el comité ético del Hospital Clínic de Barcelona. Se obtuvo por escrito el consentimiento de todos los partici- pantes, que también fueron recompensados económicamente por su participación.

\section{Diseño del estudio}

La sesión de RMf se realizó entre las 9 y las 9:30 de la mañana. Durante ese tiempo, y mediante un diseño de bloques, los participantes alternaron bloques CPT-IP y bloques control. Específicamente, después de un bloque de acomodación inicial de $35 \mathrm{~s}$, que se incluyó para hacer más familiar el escáner a los participantes, se alternaron nueve bloques CPT-IP con nueve bloques control. La tarea de CPT-IP administrada fue una modificación de la tarea de Cornblatt $[8,9]$. Concretamente, la tarea de CPT-IP que tenían que llevar a cabo los sujetos se presentaba como una serie de 27 cifras de cuatro dígitos (escogidos entre números del 1 al 9, sin repeticiones), y se les pedía que respondieran pulsando un botón tan rápido como les fuera posible, cuando se produjera la aparición de la misma cifra dos veces consecutivas. En cada bloque de CPT-IP, sólo cuatro cifras se repetían con relación al número anterior. Por su parte, la tarea de control estaba compuesta por los dígitos '1 2334 ' y fueron presentados a la misma velocidad y con los mismos intervalos que los de la tarea de CPT-IP. Las tareas de control y de CPT-IP se mostraban en bloques alternados de $20 \mathrm{~s}$, de manera que el intervalo entre estímulos era aleatorio entre $450 \mathrm{~ms}$ y $750 \mathrm{~ms}$. La duración de la adquisición completa fue de 8 min y $6 \mathrm{~s}$, durante los cuales se adquirió un total de 243 volúmenes cerebrales. Si bien con anterioridad a la sesión de RMf se había explicado en qué consistía la tarea, antes de cada bloque de CPT-IP y de control se mostraban, durante un período de $5 \mathrm{~s}$, unas breves instrucciones a cada participante. Se utilizó el programa Presentation v. 0.76 (Neurobehavioral Systems, EE. UU.) para mostrar los estímulos a los participantes. Antes de la exploración de RMf, los participantes realizaron una versión de prueba de la tarea para asegurarse de que lo habían entendido.

\section{Adquisición de imágenes}

El estudio se realizó con un escáner de RM de $3 \mathrm{~T}$ (Magnetom Trio Tim, Siemens Medical Systems, Alemania) en el Centre de Diagnòstic per la Imatge del Hospital Clínic de Barcelona. El protocolo de resonancia magnética incluye la adquisición de un conjunto de datos de RMf de 243 volúmenes de 36 cortes cada uno (usando un gradiente ecoplanarsecuencia EPI), así como un conjunto de datos 3D estructurales de alta resolución $\left(T_{1}\right.$-weighted mag- 
netization prepared rapid gradient echo - MP-RAGE image) para corregistrarlo con las imágenes de RMf. Los parámetros de adquisición para la RMf fueron: tiempo de repetición (TR): $2.000 \mathrm{~ms}$; tiempo de eco (TE): $29 \mathrm{~ms}$; porcentaje phase field of view: 100; tamaño de matriz: $128 \times 128$; grosor de corte: $3,75 \mathrm{~mm}$; espacio entre cortes: $0,75 \mathrm{~mm}$; flip: ángulo de $90^{\circ}$. Los parámetros de las imágenes estructurales fueron: TR: $2.300 \mathrm{~ms}$; TE: 2,98 ms; tiempo de inversión (TI): 900 ms; campo de visión: $256 \times 256 \mathrm{~mm}$; tamaño de matriz: $256 \times 256$; flip: ángulo de $9^{\circ}$; grosor de corte: $1 \mathrm{~mm}$.

\section{Análisis del rendimiento en la tarea de CPT-IP}

El análisis del rendimiento se realizó a partir de las siguientes variables: precisión (número de elementos identificados correctamente, aciertos), falsos positivos (número de respuestas 'sí' incorrectas, comisiones) y número de omisiones. Se calcularon también el índice de discriminación $\left(d^{\prime}\right)$ y el tiempo de reacción, este último mediante el cálculo de la media del tiempo de reacción (en milisegundos) para los estímulos target.

\section{Análisis de las imágenes de RMf}

Para el procesamiento de las imágenes se utilizó el Statistical Parametric Mapping (SPM8, Wellcome Department of Cognitive Neurology, Londres). Inicialmente se corrigieron los movimientos y se realinearon todas las imágenes para eliminar cualquier cambio menor en la señal relacionado con el movimiento. Posteriormente se normalizaron todos los volúmenes con un template EPI incluido en el SPM8. Durante la normalización espacial, todos los datos fueron remuestreados a vóxeles isotrópicos de $2 \mathrm{~mm}^{3}$. El ruido de baja frecuencia se eliminó con un filtro de paso alto (128 s) aplicado a las series de RMf en cada vóxel. Por último, las imágenes fueron suavizadas con un kernel gaussiano (FWHM) de $8 \mathrm{~mm}$ de ancho. Los análisis estadísticos se realizaron inicialmente en cada participante, para lo cual se aplicó un contraste lineal comparando la activación de los bloques de CPT-IP con la de los bloques de control, para posteriormente realizar el contraste grupal entre los dos bloques para identificar el patrón espaciotemporal de la actividad relacionada con los procesos de atención sostenida y de memoria de trabajo.

Los análisis se realizaron a nivel de todo el cerebro y los resultados fueron interpretados sólo si los valores superaban el umbral voxel-wise $(p<0,05)$ (corrección false discovery rate) (tamaño del cluster, $k \geq 15$ vóxeles). La localización anatómica de las
Figura 1. Áreas de mayor actividad cerebral en el contraste 'CPT-IP > Control'.

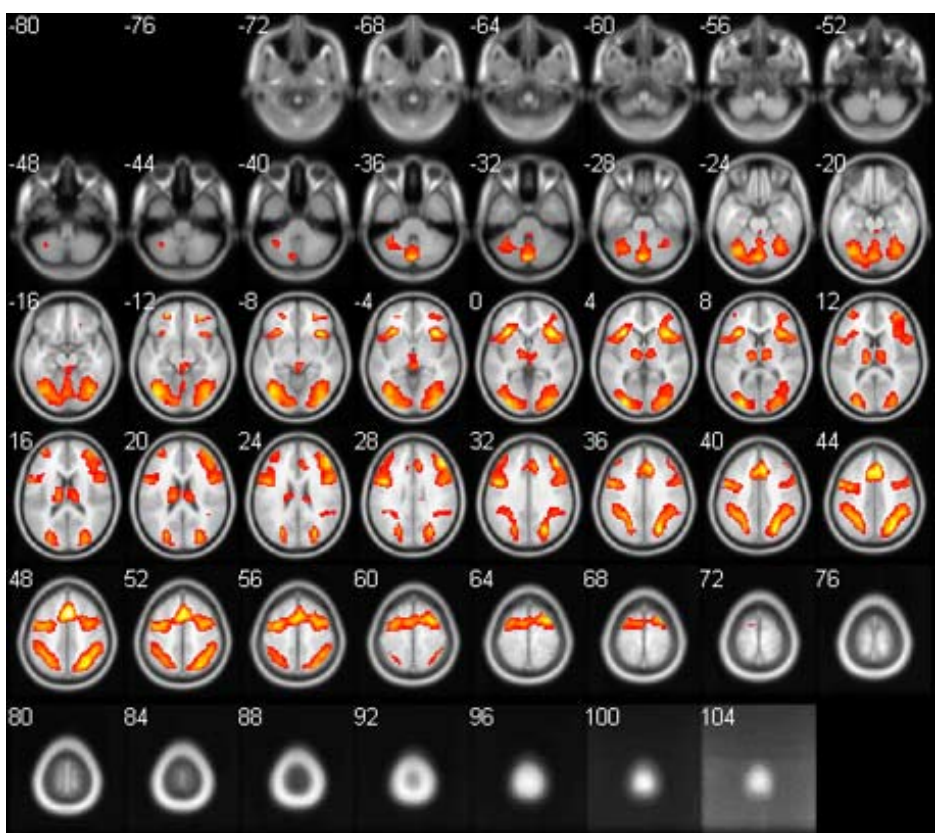

áreas cerebrales activadas se determinó mediante las coordenadas del Montreal Neurological Institute.

\section{Resultados}

\section{Análisis del rendimiento en la tarea de CPT-IP}

El rendimiento en la tarea de CPT-IP fue bueno en los 40 participantes. Grupalmente, el número de aciertos fue de media de 28,62 $\pm 4,18$ (79\% de aciertos), el número de comisiones fue de media de 6,62 \pm 3,47 , y la media de omisiones fue de $6,43 \pm 3,85$, obteniendo un índice de discriminación medio de $5,4 \pm 1,8)$. La media del tiempo de reacción fue de $503,25 \pm 119,69 \mathrm{~ms}$. No se encontraron diferencias de género en ninguna de las variables de rendimiento analizadas.

\section{Resultados de la RMf}

El contraste 'CPT-IP > Control' se calculó en todos los participantes y a nivel grupal para estudiar el patrón de activación cerebral relacionado con la atención sostenida y la memoria de trabajo. Las activaciones resultantes se localizaron en el lóbulo 
Tabla I. Áreas de mayor actividad cerebral en el contraste 'CPT-IP > Control' (las coordenadas $x, y, z$ corresponden al valor máximo de activación de cada cluster).

\begin{tabular}{|c|c|c|c|c|c|}
\hline \multirow{2}{*}{$\begin{array}{l}\text { Tamaño del } \\
\text { cluster (vóxeles) }\end{array}$} & \multicolumn{3}{|c|}{ Coordenadas (mm) } & \multirow{2}{*}{ Puntuación $T$} & \multirow{2}{*}{ Región } \\
\hline & $x$ & $y$ & $z$ & & \\
\hline \multirow{7}{*}{2.409} & 3 & 17 & 46 & 17,26 & BA 32 derecha \\
\hline & -3 & 17 & 46 & 15,16 & BA 32 izquierda \\
\hline & 45 & 35 & 31 & 13,47 & BA 9 derecha \\
\hline & -45 & 5 & 28 & 11,70 & BA 9 izquierda \\
\hline & -33 & -4 & 55 & 11,05 & BA 6 izquierda \\
\hline & 39 & 2 & 52 & 9,50 & BA 6 derecha \\
\hline & -57 & 8 & 19 & 8,35 & BA 44 izquierda \\
\hline \multirow{2}{*}{1.579} & 24 & -70 & 37 & 14,64 & BA 7 derecha \\
\hline & 42 & -43 & 46 & 11,18 & BA 40 derecha \\
\hline \multirow{6}{*}{1.788} & -27 & -88 & 7 & 11,98 & BA 19 izquierda \\
\hline & -39 & -70 & -11 & 11,55 & BA 19 izquierda \\
\hline & -39 & -46 & 46 & 11,13 & BA 40 izquierda \\
\hline & -36 & -85 & -8 & 10,50 & BA 18 izquierda \\
\hline & -27 & -55 & 43 & 9,95 & BA 7 izquierda \\
\hline & -21 & -94 & -2 & 9,93 & BA 17 izquierda \\
\hline 36 & 27 & 47 & -11 & 11,39 & BA 11 derecha \\
\hline 32 & -36 & 53 & 16 & 7,16 & BA 10 izquierda \\
\hline 18 & -24 & 50 & -11 & 10,16 & BA 11 izquierda \\
\hline
\end{tabular}

frontal -bilateralmente en las áreas de Brodmann (BA) 6 y 9, así como en las BA 10, 11 y 44 del hemisferio izquierdo-, en el lóbulo parietal (bilateralmente en las BA 7 y 40) y en la corteza occipital izquierda (BA 17 y 18), y de forma bilateral en la BA 19. Se observó también la activación bilateral del córtex cingulado anterior (BA 32) (Tabla I y Fig. 1).

El contraste 'Control > CPT-IP' mostró un patrón de activación bilateral en el lóbulo frontal (BA 4, 6,8 y 25) y del hemisferio izquierdo en las BA 9, 10, 11, 45 y 47. Asimismo, se registró activación parietal derecha (BA 2, 3, 5 y 40) y parietal izquierda (BA 7 y 39), y activación de la corteza temporal izquierda (BA 20, 21, 28, 34, 37 y 38) y derecha (BA 22, 36 y 42 ) (Tabla II y Fig. 2).

\section{Discusión}

El objetivo de nuestro estudio fue el diseño de una tarea para evaluar las regiones cerebrales asociadas con la atención sostenida y la memoria de trabajo, con la finalidad de proporcionar datos normativos en la investigación interesada en el papel de estas funciones tanto en población sana como en pacientes afectados de alteraciones de estas funciones.

El análisis del rendimiento en la tarea de CPT-IP mostró un buen nivel de rendimiento de todos los sujetos, en torno al $80 \%$. Por su parte, el análisis de los efectos relacionados con el diseño de la RMf indicó que la tarea de CPT-IP activa una red neural relacionada con la atención sostenida y la memoria de trabajo, que incluye áreas frontales, parietales y occipitales. Este patrón de activación es similar al observado previamente en la evaluación de la atención sostenida y la memoria de trabajo utilizando otros diseños [3,17,22].

La activación frontal observada incluye las áreas premotora (BA 6) y prefrontales dorsolateral (BA 9 y 10), ventral (BA 11) y ventrolateral izquierda (BA 44), así como la zona ventromedial (BA 32). El área premotora lateral es probablemente el área más activada. Sus funciones básicas se han relacionado con la secuenciación motora y la planificación de movimientos. Aunque esta activación puede estar puramente relacionada con la respuesta motora, estudios precedentes han demostrado que esta región está también involucrada en el rendimiento de la memoria de trabajo [23,24]. Las activaciones de las zonas prefrontales ventral y lateral pueden estar relacionadas con las funciones ejecutivas y la memoria de trabajo [25]. Además, la activación del área de Broca (BA 44) podría estar asociada con los procesos de la memoria de trabajo verbal involucrados en la tarea de CPT-IP, ya que se ha encontrado que esta región se activa en las tareas que requieren altas demandas de memoria de trabajo verbal $[23,24]$. Por su parte, la corteza cingulada anterior (BA 32) se ha asociado a la realización de tareas cognitivas de monitorización de conflictos, y es un factor importante para facilitar la detección del estímulo apropiado, mientras se ignoran los estímulos irrelevantes. Por otra parte, también se ha asociado con la detección de errores y de respuesta inmediata de reajuste [26-28], en cooperación con las áreas prefrontales dorsolateral y ventral. Asimismo, la corteza cingulada anterior se ha asociado con el mantenimiento de los números en la memoria de trabajo, facilitando así las operaciones mentales [29]. Ello podría explicar las activaciones encontradas en esta región en el presente trabajo, ya que, en nuestra ta- 
rea de CPT-IP, era necesario el mantenimiento de los números en la memoria de trabajo, al tratarse de una tarea $n$-back.

$\mathrm{Al}$ igual que en estudios anteriores, nuestra tarea de CPT-IP activó bilateralmente regiones parietales. La corteza parietal participa de la alerta y la reorientación de la atención [2,30]. También se le asocian funciones ejecutivas, como la asignación de los procesos de atención y memoria de trabajo verbal, la mediación del almacenamiento a corto plazo y la recuperación de material verbal codificado fonológicamente [31]. Por consiguiente, las regiones parietales podrían contribuir al mantenimiento de las cifras en la memoria de trabajo verbal $[6,32]$.

Otro cluster significativo de activaciones se encuentra en la corteza occipital, tanto en las zonas primarias como en las asociativas. Como han puesto de manifiesto estudios anteriores [17], la activación occipital puede reflejar procesos de análisis e identificación de los estímulos presentados visualmente. En concreto, en nuestro caso puede estar reflejando el procesamiento numérico asociado a la tarea de CPT-IP. En este sentido, se ha observado que la corteza visual asociativa contribuye a la identificación de los números [33,34], además de las letras [35], los objetos [36] y el reconocimiento de rostros [37]. En la tarea de control, la activación de regiones visuales fue menor, posiblemente porque el reconocimiento era más fácil debido a que el estímulo fue siempre el mismo ('1 2234 ').

Cabe destacar que el contraste 'Control > CPT-IP' mostró activaciones en diferentes áreas cerebrales (Fig. 2 y Tabla II), las cuales podrían estar relacionadas con la default mode network. Específicamente, se encontraron activaciones relacionadas con la tarea de 'Control' en las cortezas motora primaria y secundaria, las cortezas prefrontales dorsolateral, anterior y orbitofrontal, la corteza subgenual, la circunvolución inferior prefrontal, las cortezas somatosensorial primaria y de asociación, la circunvolución angular, el giro supramarginal, así como en diferentes regiones del lóbulo temporal. Estas áreas tendrían menos requisitos metabólicos en estado de reposo $[17,22]$ y se desactivarían cuando se necesita un esfuerzo atencional a los estímulos externos [38]. La importancia de su desactivación aún no está del todo clara, aunque se ha sugerido que podría reflejar una inhibición de los procesos que interfieren en la correcta ejecución de la tarea, como el seguimiento externo e interno de los estímulos. En este sentido, la desactivación encontrada en nuestro estudio podría representar un proceso de optimización en tareas que exigen una gran cantidad de recursos atencionales $[39,40]$.
Tabla II. Áreas de mayor actividad cerebral en el contraste 'Control > CPT-IP' (las coordenadas $x, y, z$ corresponden al valor máximo de activación de cada cluster).

\begin{tabular}{|c|c|c|c|c|c|}
\hline \multirow{2}{*}{$\begin{array}{l}\text { Tamaño del } \\
\text { cluster (vóxeles) }\end{array}$} & \multicolumn{3}{|c|}{ Coordenadas (mm) } & \multirow{2}{*}{ Puntuación $T$} & \multirow{2}{*}{ Región } \\
\hline & $x$ & $y$ & $z$ & & \\
\hline \multirow{6}{*}{2.317} & 24 & -37 & 67 & 9,73 & BA 2 derecha \\
\hline & 21 & -40 & 73 & 9,62 & BA 5 derecha \\
\hline & 3 & -19 & 52 & 8,93 & BA 6 derecha \\
\hline & -3 & -52 & 64 & 7,87 & BA 7 izquierda \\
\hline & -21 & -34 & 64 & 7,54 & BA 3 izquierda \\
\hline & 42 & -22 & 64 & 6,72 & BA 4 derecha \\
\hline \multirow{12}{*}{2.733} & 3 & 59 & -5 & 17,56 & BA 10 derecha \\
\hline & 3 & 38 & -14 & 15,86 & BA 11 derecha \\
\hline & -21 & 35 & 46 & 15,61 & BA 8 izquierda \\
\hline & -6 & 47 & -8 & 15,54 & BA 10 izquierda \\
\hline & -54 & -7 & -23 & 13,63 & BA 20 izquierda \\
\hline & -27 & -37 & -11 & 12,28 & BA 37 izquierda \\
\hline & -3 & 56 & 31 & 10,99 & BA 9 izquierda \\
\hline & -21 & -13 & -17 & 10,23 & BA 28 izquierda \\
\hline & -15 & -4 & -20 & 9,89 & BA 34 izquierda \\
\hline & 3 & 8 & -5 & 9,60 & BA 25 derecha \\
\hline & -39 & 14 & -32 & 8,32 & BA 38 izquierda \\
\hline & -48 & 8 & -32 & 7,95 & BA 21 izquierda \\
\hline \multirow{6}{*}{769} & 51 & 2 & -23 & 8,91 & BA 21 derecha \\
\hline & 42 & 8 & -29 & 8,47 & BA 38 derecha \\
\hline & 60 & -4 & 4 & 8,42 & BA 22 derecha \\
\hline & 51 & -22 & 13 & 7,74 & BA 40 derecha \\
\hline & 27 & -37 & -8 & 7,00 & BA 36 derecha \\
\hline & 66 & -28 & 7 & 5,53 & BA 42 derecha \\
\hline 251 & -42 & -73 & 31 & 14,55 & BA 39 izquierda \\
\hline 98 & 48 & -70 & 31 & 13,56 & BA 39 derecha \\
\hline \multirow{2}{*}{54} & -30 & 32 & -17 & 7,76 & BA 47 izquierda \\
\hline & -33 & 35 & -14 & 7,76 & BA 11 izquierda \\
\hline 21 & -51 & 32 & 4 & 6,61 & BA 45 izquierda \\
\hline
\end{tabular}


Figura 2. Áreas de mayor actividad cerebral en el contraste 'Control > CPT-IP'.

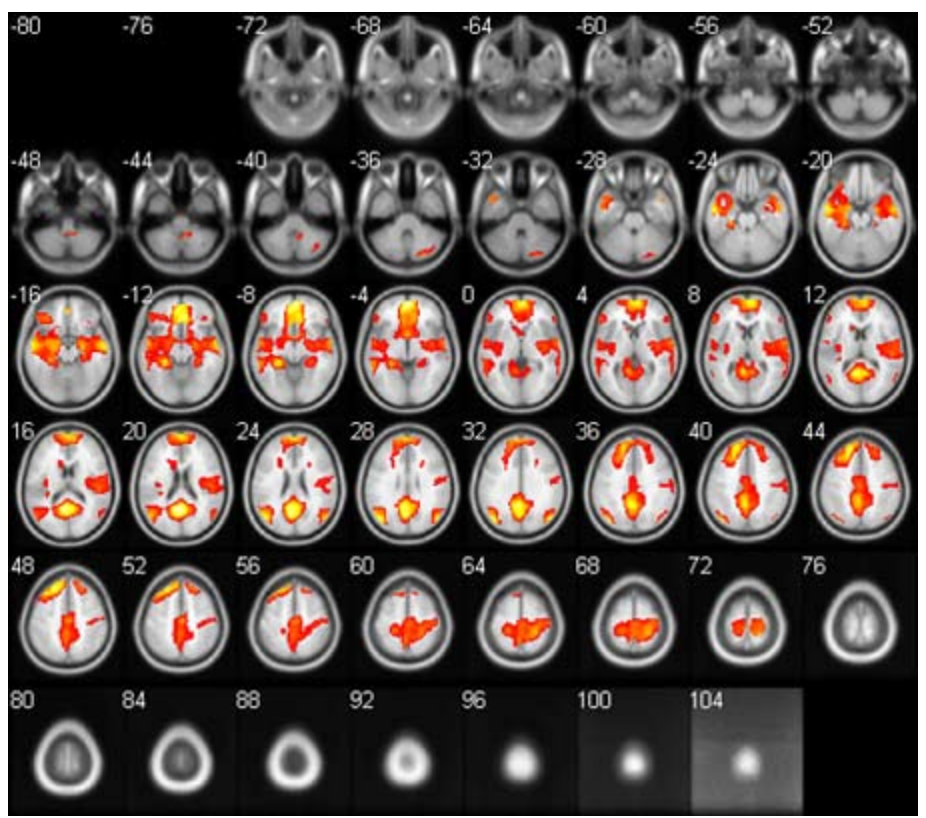

Nuestro estudio no está exento de algunas limitaciones. En primer lugar, si se hubieran incluido diferentes niveles de dificultad en la tarea de CPTIP, probablemente ésta hubiera sido más sensible y podían haberse observado diferencias en el patrón de activación. En segundo lugar, el uso de análisis multivariantes podría contribuir a delinear mejor el sustrato neural de la atención sostenida y la memoria de trabajo.

Finalmente, este estudio proporciona datos normativos que podrían ser utilizados para la investigación relacionada con el papel de la atención y la memoria de trabajo en diferentes trastornos psicopatológicos y del neurodesarrollo. En este sentido, la tarea de CPT se ha usado para estudiar la enfermedad de Alzheimer [10,11], la enfermedad de Parkinson [12], la discalculia $[13,14]$ y el TDAH $[15,16]$. En cuanto a la psicopatología, un diseño similar se ha utilizado para estudiar el trastorno bipolar [9]. Con relación a ello, nuestro estudio parece más robusto, al haber utilizado una muestra más grande y más homogénea en términos de edad y género que en investigaciones anteriores. Por otro lado, en relación con el estudio de los trastornos del neurodesarrollo, el CPT-IP podría utilizarse para estudiar la discalculia. Este trastorno del aprendizaje se carac- teriza por dificultades en la atención y en la memoria de trabajo $[41,42]$ y tiene una alta comorbilidad con el TDAH, lo que a veces hace difícil su diagnóstico diferencial. En la actualidad no existen estudios centrados en el sustrato neural de estos déficits en la discalculia. Por tanto, el uso de un enfoque de RMf puede contribuir a delimitar las correlaciones cerebrales funcionales de los déficits de atención y de memoria de trabajo característicos de la discalculia.

En conclusión, la tarea de CPT-IP utilizada en nuestro estudio se asoció a un patrón de activación cerebral caracterizado por activaciones relacionadas con la atención sostenida y la memoria de trabajo, el cual implica una red formada por regiones frontales, parietales y occipitales. Los datos obtenidos pueden considerarse como normativos y sugieren que la tarea de CPT-IP desarrollada podría ser una buena herramienta para evaluar las dificultades de atención y de memoria de trabajo en diferentes trastornos, como la discalculia.

\section{Bibliografía}

1. Posner MI. Imaging attention networks. Neuroimage 2012; 61: 450-6.

2. Raz A, Buhle J. Typologies of attentional networks. Nature Rev Neurosci 2006; 7: 367-79.

3. Fan J, McCandliss BD, Fossella J, Flombaum JI, Posner MI. The activation of attentional networks. Neuroimage 2005; 26: 471-9.

4. Cabeza R, Nyberg L. Imaging cognition: II. An empirical review of 275 PET and fMRI studies. J Cogn Neurosci 2000; 11: 80-93.

5. Coull JT. Neural correlates of attention and arousal: insights from electrophysiology, functional neuroimaging and psychopharmacology. Prog Neurobiol 1998; 55: 343-61.

6. Lepsien J, Thornton I, Nobre AC. Modulation of workingmemory maintenance by directed attention. Neuropsychologia 2011; 49: 1569-77.

7. Hirata A, Castro-Alamancos MA. Neocortex network activation and deactivation states controlled by the thalamus. J Neurophysiol 2010; 103: 1147-57.

8. Cornblatt BA, Lezenweger MF, Erlenmeyer-Kimling L. The Continuous Performance Test, Identical Pairs Version: II. Contrasting attentional profiles in schizophrenic and depressed patients. Psychiatry Res 1989; 29: 65-85.

9. Strakowski SM, Adler CM, Holland SK, Mills N, DelBello MP. A preliminary FMRI study of sustained attention in euthymic, unmedicated bipolar disorder. Neuropsychopharmacology 2004; 29: 1734-40.

10. White HK, Levin ED. Four-week nicotine skin patch treatment effects on cognitive performance in Alzheimer's disease. Psychopharmacology 1999; 143: 158-65.

11. Estévez-González A, García-Sánchez C, Boltes A, GarcíaNonell C, Rigau-Ratera E, Otermín P, et al. Atención sostenida en la fase preclínica de la enfermedad de Alzheimer. Rev Neurol 2003; 36: 829-32.

12. Kelton MC, Kahn HJ, Conrath CL, Newhouse P. The effects of nicotine on Parkinson's disease. Brain Cogn 2000; 43: 274-82.

13. Lindsay RL, Tomazic T, Levine MD, Accardo PJ. Attentional function as measured by a Continuous Performance Task in children with dyscalculia. J Dev Behav Pediatr 2001; 42: 1049-56.

14. Miranda-Casas A, Meliá-De Alba A, Marco-Taverner R, Roselló B, Mulas F. Dificultades en el aprendizaje de matemáticas 
en niños con trastorno por déficit de atención e hiperactividad. Rev Neurol 2006; 42 (Supl 2): S163-70.

15. Groom MJ, Jackson GM, Calton TG, Andrews HK, Bates AT, Liddle PF, et al. Cognitive deficits in early-onset schizophrenia spectrum patients and their non-psychotic siblings: a comparison with ADHD. Schizophr Res 2008; 99: 85-95.

16. Fernández-Jaén A, Martín Fernández-Mayoralas D, CallejaPérez B, Moreno-Acero N, Muñoz-Jareño N. Efectos del metilfenidato en los procesos cognitivo-atencionales. Uso de los test de ejecución continuada. Rev Neurol 2008; 46 (Supl 1): S47-9.

17. Casey BJ, Formans SD, Franzen P, Berkowitz A, Braver TS, Nystrom LE, et al. Sensitivity of prefrontal cortex to changes in target probability: a functional MRI study. Hum Brain Mapp 2001; 13: 26-33.

18. Ogg RJ, Zou P, Allen DN, Hutchins SB, Dutkiewicz RM, Mulhern RK. Neural correlates of a clinical continuous performance test. Magn Reson Imaging 2008; 26: 504-12.

19. Keilp JG, Herrera J, Stritzke P, Cornblatt BA. The Continuous Performance Test, Identical Pairs version (CPT-IP): III. Brain functioning during performance of numbers and shapes subtasks. Psychiatry Res 1997; 74: 35-45.

20. Adan A, Serra-Grabulosa JM. Effects of caffeine and glucose, alone and combined, on cognitive performance. Hum Psychopharmacol 2010; 25: 310-7.

21. Serra-Grabulosa JM, Adán A, Falcón C, Bargalló N. Glucose and caffeine effects on sustained attention: an exploratory fMRI study. Hum Psychopharmacol Clin Exper 2010; 25: 310-7.

22. Lawrence NS, Ross TJ, Hoffman R, Garavan H, Stein EA. Multiple neuronal networks mediate sustained attention. J Cogn Neurosci 2003; 15: 1028-38.

23. Newhart M, Trupe LA, Gomez Y, Cloutman L, Molitoris JJ, Davis CC, et al. Asyntactic comprehension, working memory, and acute ischemia in Broca's area versus angular gyrus. Cortex 2012; 48: 1288-97.

24. Ranganath C, Johnson MK, D'Esposito M. Prefrontal activity associated with working memory and episodic long-term memory. Neuropsychologia 2003; 41: 378-89.

25. D’Esposito M. Working memory. In Goldenberg G, Miller B, eds. Handbook of clinical neurology: neuropsychology and behavioral neurology. London: Elsevier; 2008. p. 237-47.

26. Grodzinsky Y, Santi A. The battle for Broca's region. Trends Cogn Sci 2008; 12: 474-80.

27. Bediou B, Koban L, Rosset S, Pourtois G, Sander D. Delayed monitoring of accuracy errors compared to commission errors in ACC. Neuroimage 2012; 60:1925-36.
28. Ridderinkhof KR, Ullsperger M, Crone EA, Nieuwenhuis S. The role of the medial frontal cortex in cognitive control. Science 2004; 306: 443-7.

29. Zago L, Petit L, Turbelin MR, Andersson F, Vigneau M, Tzourio-Mazoyer N. How verbal and spatial manipulation networks contribute to calculation: an fMRI study. Neuropsychologia 2008; 46: 2403-14.

30. Konrad K, Neufang S, Thiel CM, Specht K, Hanisch C, Fan J, et al. Development of attentional networks: an fMRI study with children and adults. Neuroimage 2005; 28: 429-39.

31. Jonides J, Schumacher EH, Smith EE, Koeppe RA, Awh E, Reuter-Lorentz PA, et al. The role of parietal cortex in verbal working memory. J Neurosci 1998; 18: 5026-34.

32. Coull JT, Frith CD, Frackowiak RSJ, Grasby PM. A frontoparietal network for rapid visual information processing: a PET study of sustained attention and working memory Neuropsychologia 1996; 34: 1085-95.

33. Dehaene S, Molko N, Cohen L, Wilson AJ. Arithmetic and the brain. Curr Opin Neurobiol 2004; 14: 218-24.

34. Price GR, Ansari D. Symbol processing in the left angular gyrus: evidence from passive perception of digits. Neuroimage 2011; 57: 1205-11.

35. Szwed $\mathrm{M}$, Dehaene $\mathrm{S}$, Kleinschmidt $\mathrm{A}$, Eger $\mathrm{E}$, Valabrègue $\mathrm{R}$, Amadon A, et al. Specialization for written words over objects in the visual cortex. Neuroimage 2011; 56: 330-44.

36. Grill-Spector K, Kourtzi Z, Kanwisher N. The lateral occipital complex and its role in object recognition. Vision Res 2001; 41: 1409-22.

37. Minnebusch DA, Suchan B, Köster O, Daum I. A bilateral occipitotemporal network mediates face perception. Behav Brain Res 2009; 198: 179-85.

38. Raichle ME, MacLeod AM, Snyder AZ, Powers WJ, Gusnard DA, Shulman GL. A default mode of brain function. Proc Natl Acad Sci U S A 2001; 98: 676-82.

39. Gusnard DA, Raichle ME. Searching for a baseline: functional imaging and the resting human brain. Nat Rev Neurosci 2001; 2: 685-94.

40. Mayer JS, Roebroeck A, Maurer K, Linden DE. Specialization in the default mode: task-induced brain deactivations dissociate between visual working memory and attention. Hum Brain Mapp 2010; 31: 126-39.

41. Askenazi S, Henik A. Attentional networks in developmental dyscalculia. Behav Brain Func 2010; 6: 2.

42. Passolunghi MC, Siegel LS. Short-term memory, working memory, and inhibitory control in children with difficulties in arithmetic problem solving. J Exp Child Psychol 2001; 80: 44-57.

Cerebral networks of sustained attention and working memory: a functional magnetic resonance imaging study based on the Continuous Performance Test

Introduction. One of the most used paradigms in the study of attention is the Continuous Performance Test (CPT). The identical pairs version (CPT-IP) has been widely used to evaluate attention deficits in developmental, neurological and psychiatric disorders. However, the specific locations and the relative distribution of brain activation in networks identified with functional imaging, varies significantly with differences in task design.

Aim. To design a task to evaluate sustained attention using functional magnetic resonance imaging (fMRI), and thus to provide data for research concerned with the role of these functions.

Subjects and methods. Forty right-handed, healthy students (50\% women; age range: $18-25$ years) were recruited. A CPT-IP implemented as a block design was used to assess sustained attention during the fMRI session.

Results. The behavioural results from the CPT-IP task showed a good performance in all subjects, higher than $80 \%$ of hits. fMRI results showed that the used CPT-IP task activates a network of frontal, parietal and occipital areas, and that these are related to executive and attentional functions.

Conclusions. In relation to the use of the CPT to study of attention and working memory, this task provides normative data in healthy adults, and it could be useful to evaluate disorders which have attentional and working memory deficits.

Key words. CPT-IP. fMRI. Parietal cortex. Prefrontal cortex. Sustained attention. Working memory. 